\title{
Fatal Pulmonary Embolus After Uterine Artery Fibroid Embolisation
}

\author{
Haitham Hamoda $\cdot$ P. Tait $\cdot$ D. K. Edmonds
}

Received: 1 March 2009/ Accepted: 2 April 2009/Published online: 16 May 2009

(C) Springer Science+Business Media, LLC and the Cardiovascular and Interventional Radiological Society of Europe (CIRSE) 2009

\begin{abstract}
We report a 44-year-old woman who developed a fatal pulmonary embolus after uterine artery fibroid embolisation (UAE). Bilateral UAE was carried out through a single right-femoral artery puncture. The largest fibroid in the anterior fundal wall measured $4.5 \mathrm{~cm}$, and the largest fibroid in the posterior fundal wall measured $6 \mathrm{~cm}$. The appearances after UAE were satisfactory, and the procedure was apparently uneventful. No immediate complications were noted. The patient developed sudden-onset shortness of breath and went into cardiac arrest $19 \mathrm{~h}$ after the procedure. Postmortem autopsy confirmed that the cause of a death was a pulmonary embolism. To our knowledge this is the first reported case in the United Kingdom in which death occurred from a pulmonary embolus after UAE.
\end{abstract}

Keywords Fibroid embolisation - Complications · Interventional radiology

\section{Introduction}

Uterine artery fibroid embolisation (UAE) has now been used in clinical practice for $>2$ decades in the management of symptomatic uterine fibroids [1, 2]. It is estimated that $>50,000$ procedures have been performed worldwide since Ravina reported his series in France in 1995 [3, 4].

H. Hamoda $(\varangle) \cdot$ D. K. Edmonds

Queen Charlotte's Hospital, London, UK

e-mail: haithamhamoda@hotmail.com

P. Tait

Hammersmith Hospital, London, UK
UAE is emerging as an effective alternative to hysterectomy and myomectomy in women with fibroids. Although long-term follow up data are still lacking, early reports regarding its safety and efficacy have been encouraging, and the procedure is increasingly being accepted [1]. However, there is a lack of evidence on future fertility and pregnancy outcomes in women undergoing UAE [5]. We report here a case of death from a pulmonary embolus after UAE.

\section{Case Report}

A 44-year-old nulliparous woman with a body mass index of 27 underwent UAE in July 2008 for symptomatic relief of heavy menstrual bleeding. She had previously undergone endoscopic resection of a submucous fibroid in 2002 and open myomectomy in 2003. She subsequently underwent exploratory laparotomy and division of adhesions for relief of small-bowel obstruction in February 2007. A subsequent hysteroscopy in November 2007 showed a large cavity distorted by fibroids. A pelvic ultrasound scan before her procedure showed an antiverted, enlarged uterus measuring approximately $13.5 \times 8 \times 9 \mathrm{~cm}$. The myometrium was noted to be diffusely heterogenous, containing multiple fibroids in the anterior and posterior walls. The largest fibroid measured $6 \mathrm{~cm}$. Bilateral UAE was carried out through a single right-femoral artery puncture using a $5 \mathrm{~F}$ sheath, a $4 \mathrm{~F}$ catheter, and a $3 \mathrm{~F}$ coaxial catheter. Multiple injections of contrast were administered into the internal iliac arteries and uterine arteries. The uterus was noted to be hypervascular and supplied by hypertrophied uterine arteries. Each uterine artery was selectively catheterised and embolised with polyvinyl alcohol particles (William Cook Europe, Bjaeverskov, Denmark). One vial 
of particles $350-500 \mu \mathrm{m}$ in diameter and two vials of particles $510-700 \mu \mathrm{m}$ in diameter were used. The end point of the embolisation process was near-stasis in the uterine artery. The appearances after UAE were satisfactory. The procedure lasted approximately $40 \mathrm{~min}$ and was apparently uneventful. No immediate complications were noted.

The patient was admitted for observation overnight. The plan was for her to be on bed rest for $12 \mathrm{~h}$ for observation of the puncture site for bleeding or swelling and administration of analgesia (diclofenac sodium and paracetamol).

Nineteen hours after the procedure, the patient had sudden-onset shortness of breath and collapsed. She was noted to be semicomatose with a Glasgow Coma Scale score of eight. She then had respiratory arrest, followed by cardiac arrest. She received cardiopulmonary resuscitation (CPR) for $>1 \mathrm{~h}$. However, there was no cardiac activity throughout CPR, and she was noted to have pulseless electrical activity on cardiac monitoring. CPR was discontinued after $1 \mathrm{~h}$ and $10 \mathrm{~min}$, and the patient was pronounced dead. Postmortem autopsy confirmed that the cause of a death was pulmonary embolism.

\section{Discussion}

To our knowledge this is the first reported case in the United Kingdom in which death occurred from pulmonary embolisation after UAE. The embolisation procedure had appeared routine. The embolic agents used and the quantity thereof were not unusual. The size of the uterus was not considered likely to have caused compression and stasis in the lower-limb venous structures. Certainly, patients with symptoms from a uterus of greater size than that seen in this patient have been treated at our institution. It is not clear from the postmortem autopsy if the origin of the embolus was from the pelvic or the lower-limb veins. Theoretically, it is possible to surmise that pelvic veins are enlarged and engorged in the presence of multiple hyper vascular fibroids, which consequently have decreased flow after embolisation, thus producing a potential scenario for thrombosis. Relative contrast stasis can be visualised in veins draining the uterus during any embolisation procedure. The patient was kept for overnight observation per hospital policy. If the treatment had been carried out as a day case, then death would have occurred at home. The precipitous nature of the events raises the question as to whether there was a pre-existing thrombosis, which may have rapidly progressed after UAE. There was a history of previous pelvic surgery; however, there was no clinical evidence for pelvic or lower-limb venous thrombosis or clinical history of thrombotic tendency before the embolisation procedure was performed.
At least five deaths have been reported after UAE. Two women died after UAE from septic shock, one in the United Kingdom [6] and the other in The Netherlands [7]. One woman with known breast cancer died in Italy several days after UAE from a pulmonary embolus [8], and two women died in the United States from pulmonary emboli after the procedure [5].

Although the risk of developing a fatal venous thromboembolus is considered low, there have so far been at least four reported cases in the literature. We advocate that women undergoing this procedure should routinely receive thromboprophylaxis in the form of thromboembolicdeterrent stockings. However, these would be of no benefit from an embolus originating in the pelvic veins. Further research is needed to assess the role of thromboprophylaxis using heparin-like agents with this procedure. At present, there is no evidence that their use would be of benefit. Indeed, the use of such agents to decrease the incidence of embolic events (which are already rare relative to the number of procedures performed) may be accompanied by increased bleeding complications or more ineffective embolisation procedures (and therefore poor clinical outcomes) that rely on inducing local vessel thrombosis as well as physical occlusion. It is not possible to say if patients with a previous history of pelvic surgery are at greater risk of embolus after UAE.

The continued reporting of all UAE cases to a National Registry would allow us to monitor the number of women undergoing the procedure, the number and type of complications, and the fertility outcomes in all such cases.

\section{References}

1. The National Institute for Health and Clinical Excellence (2004) Systematic review of the efficacy and safety of uterine artery embolisation in the treatment of fibroids. Interventional Procedures Guidelines

2. Royal College of Radiologists and Royal College of Obstetricians and Gynaecologists (2000) Clinical recommendations on the use of uterine artery embolisation in the management of fibroids. Report of a Joint Working Party

3. Ravina JH, Bouret JM, Fried D, Benifla JL, Daraï E, Pennéhouat G, Madelenat P, Herbreteau D, Houdard E, Merland JJ (1995) Value of preoperative embolization of uterine fibroma: report of a multicenter series of 31 cases. Contracept Fertil Sex 23:45-49

4. Ravina JH, Herbreteau D, Ciraru-Vigneron N, Bouret JM, Houdart E, Aymard A, Merland JJ (1995) Arterial embolisation to treat uterine myomata. Lancet 346(8976):671-672

5. Lefebvre G, Vilos G, Asch M, Society of Obstetricians and Gynaecologists of Canada, Canadian Association of Radiologists, Canadian Interventional Radiology Association (2004) Uterine fibroid embolization (UFE). J Obstet Gynaecol Can 26(899911):913-928

6. Vashisht A, Studd J, Carey A, Burn P (1999) Fatal septicaemia after fibroid embolisation. Lancet 354(9175):307-308 
7. de Blok S, de Vries C, Prinssen HM, Blaauwgeers HL, JornaMeijer LB (2003) Fatal sepsis after uterine artery embolization with microspheres. J Vasc Interv Radiol 14:779-783

8. Lanocita R, Frigerio L, Patelli G, Di Tolla G, Spreafico C (1999) A fatal complication of percutaneous transcatheter embolization for treatment of uterine fibroids. Second International Symposium on the Embolization of Uterine Myomata, Boston, MA 